\title{
Increasing incidence and mortality from myocardial infarction in Stockholm county
}

\author{
LARS ALFREDSSON, ANDERS AHLBOM
}

\begin{abstract}
A study was undertaken to examine trends in the incidence and mortality of myocardial infarction in Sweden. All cases $(n=19908)$ of myocardial infarction diagnosed in the population of Stockholm county during 1974-80 were identified by means of the cause of death register and the inpatient care register. Information on patients at risk was obtained from the civil registration system. The relative risk of developing, or dying of, myocardial infarction in one specific year, compared with the average for the whole period, was taken as the basis for describing the trends. For men in Stockholm the incidence as well as the mortality was appreciably increased; the annual increase in incidence was $3 \%$ and in mortality 4\%. There were no signs of decreasing lethality. For women there was an appreciable increase in incidence; for mortality the result was less specific but was compatible with an increase. The observed increases in incidence and mortality appeared to be real and were probably not due to an increasing tendency for patients to seek hospital treatment or for doctors to make the diagnosis. The reason for the increase is unknown.
\end{abstract}

\section{Introduction}

Mortality from ischaemic heart disease has declined since the mid 1960s in the United States and several other industrialised countries. ${ }^{1-3}$ It is still unclear whether the decline in ischaemic heart disease stems from declining incidence or from declining lethality. ${ }^{4}$

In Sweden the morbidity as well as the mortality from this disease are low compared with many other countries, including the United States. ${ }^{56}$ The aim of the present study was to examine the trends in incidence of as well as in mortality from acute myocardial infarction in Stockholm county for the years 1974-80.

\section{Patients and methods}

Sweden has a highly efficient civil registration system. On the basis of this system the national cause of death register and the medical information systems in the county councils, which contain comprehensive inpatient care registers, have been developed.

\section{CASE SELECTION}

To find cases of myocardial infarction that had occurred in Stockholm county during the study period information was selected from the registers. From the inpatient care register were selected all hospital admissions with a diagnosis of definite myocardial infarction (Nordic ver-

Department of Social Medicine, Huddinge University Hospital, Karolinska Institute, Huddinge, Sweden

LARS ALFREDSSON, BA, research assistant

ANDERS AHLBOM, PHD, assistant professor

Corresbondence and renrint requests to: $\mathrm{Mr} \mathrm{I}$ ars Alfredsson. sion of International Classification of Diseases code 410.00 or $410.99^{7}$ ) in patients in the 40-74 year age group who were residents in Stockholm county and were discharged from hospital during 1974-80. From the cause of death register were selected all deaths that had occurred among residents of Stockholm county in the 40-74 year age group during 1974-80 in whom the underlying or one of the contributing causes of death was myocardial infarction (International Classification of Diseases code $410.0,410.9$ ). Cases of myocardial infarction were defined from these two sets of data. For patients with more than one event only those with events occurring at least 28 days after a previous one were considered as new cases in accordance with the World Health Organisation rule. ${ }^{6}$ Details of the problems and methodology connected with the use of these registers in the epidemiology of myocardial infarction have been presented elsewhere. ${ }^{89}$

During the period 1974-80 a total of 19908 cases of myocardial infarction in the 40-74 year age group were recorded. Of these, 14576 were men (median age 64 years) and 5332 were women (median age 68 years). The total number of deaths from myocardial infarction during the period was 5270 and 1965 for men and women respectively. The table shows the total number of cases of and deaths due to myocardial infarction by year of occurrence.Using the civil registration system the population at risk was calculated for each of the seven years $1974-80$ by sex and age.

Number of cases of and deaths due to myocardial infarction by year and sex

\begin{tabular}{cccccc}
\hline & \multicolumn{3}{c}{ Cases } & & \multicolumn{3}{c}{ Deaths } \\
\cline { 2 - 3 } \cline { 5 - 6 } Year & $M$ & F & & M & F \\
\hline 1974 & 1889 & 714 & & 663 & 255 \\
1975 & 1841 & 709 & & 657 & 270 \\
1976 & 2049 & 735 & & 739 & 278 \\
1977 & 2097 & 702 & & 754 & 256 \\
1978 & 2123 & 764 & & 755 & 279 \\
1979 & 2251 & 860 & & 825 & 332 \\
1980 & 2326 & 848 & & 877 & 295 \\
\hline Total & 14576 & 5332 & & 5270 & 1965 \\
\hline
\end{tabular}

STATISTICAL ANALYSIS

In the analysis men and women were treated separately. A multiplicative model was used to control the changes in the age distribution during the study. ${ }^{10}$ This model implies that the rate in one age group for one year is defined as a product of an age factor which is the same for all years and a time factor which may differ between the years but is the same in all age groups. The time factor may be interpreted as the relative risk of developing (or dying of) myocardial infarction in one specific year compared with the average over the whole period. Maximum likelihood estimates were obtained by an iterative solution of the system of likelihood equations. To investigate the adequacy of the model the individual residuals were examined and a likelihood ratio test performed. The model seemed to fit the data and in all cases the results were insignificant (figs 1-4). An overall likelihood ratio test of the hypothesis that all relative risks were equal was performed. Confidence intervals of $95 \%$ were computed for the relative risks. Regression lines were fitted to the data to describe the trends. ${ }^{11}$

\section{Results}

Fig 1 shows the relative risk for men (40-74 years) of developing myocardial infarction for each specific year. The dots indicate the point estimates of relative risk according to the multiplicative model. The value 1.00 represents the average risk for the period. Confidence intervals ot $95 \%$ are marked. 
The likelihood ratio test showed that the relative risk differed significantly between the years $\left(p=2 \times 10^{9}\right)$. Fig 1 also shows the regression line fitted to the relative risks. The slope of the line indicates that the incidence of myocardial infarction increased by about $3 \%$ per year.

Fig 2 shows the corresponding relative risks of women. These data also show a significant difference between the years and a clear increase in the relative risks during the period.

Figs 3 and 4 show the mortality data for men and women respectively. For men a significant difference in mortality from myocardial infarction was observed. According to the slope of the regression line the increase was about $4 \%$ a year. For women the mortality did not differ significantly between the years, even though the regression line showed a moderate increase.

\section{Discussion}

The results of this study of myocardial infarction in Stockholm county during the years $1974-80$ indicate that for men there were definite increases in both incidence and mortality. For women there was a definite increase in incidence; for mortality the result was less specific but was compatible with an increase.

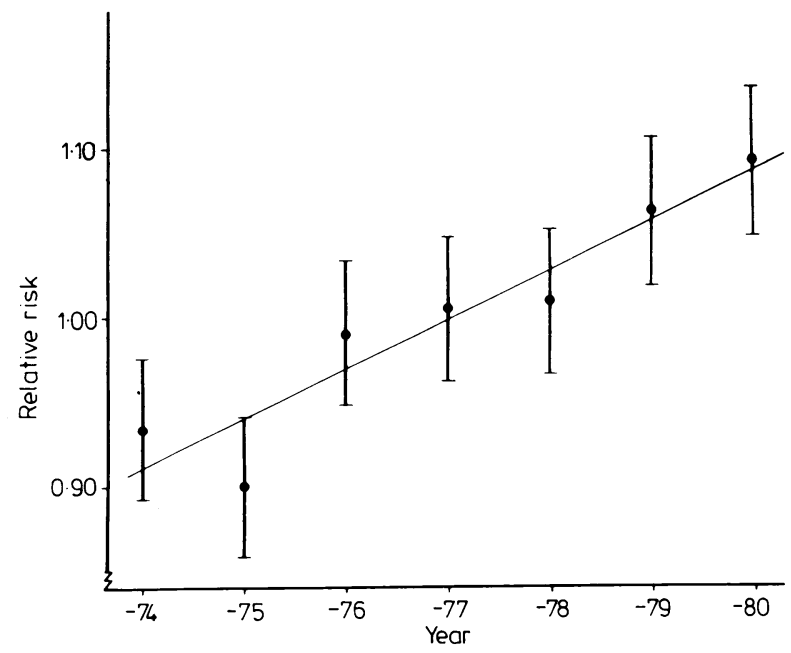

FIG 1-Incidence of myocardial infarction among men aged 40-74. Relative risks for specific years according to the multiplicative model $(1.00$ is the average for the complete period) and regression line. Test of model: $p=0 \cdot 22$. Significance test of equality between relative risk values: $p=2.0 \times 10^{-9}$ Annual increase according to regression line, $2 \cdot 9 \%$.

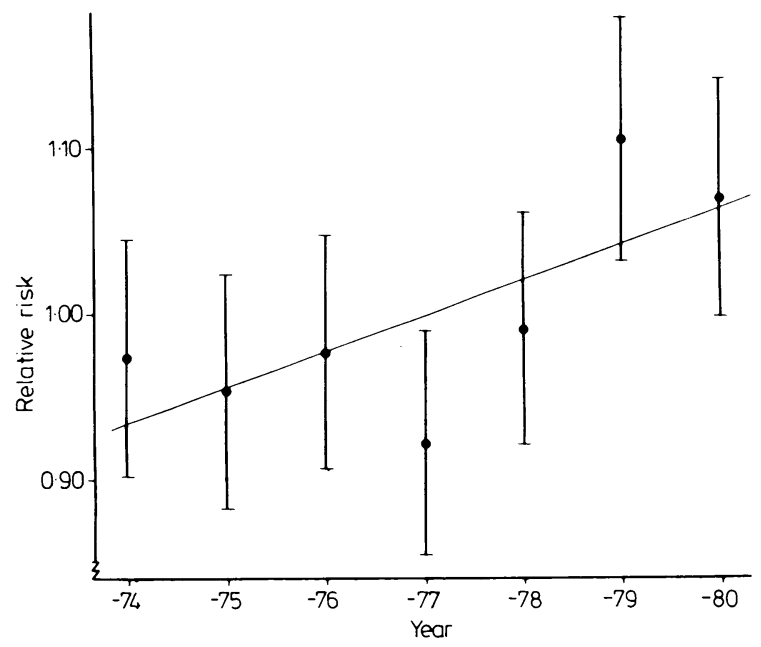

FIG 2-Incidence of myocardial infarction among women aged 40-74. Relative risks for specific years according to the multiplicative model and regression line. Test of model: $p=0 \cdot 24$. Significance test of equality between relative risk values: $p=0.003$. Annual increase according to regression line, $2 \cdot 2 \%$.

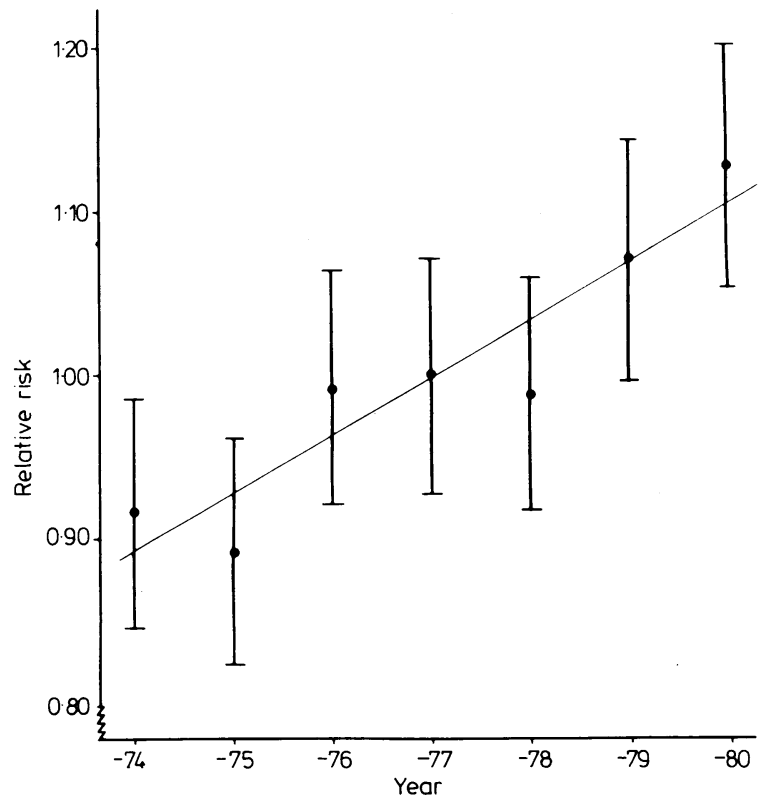

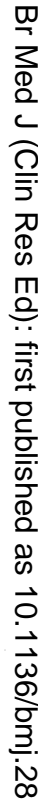

FIG 3-Mortality from myocardial infarction among men aged 40-74. Relative risks for specific years according to the multiplicative model andW regression line. Test of model : $p=0.45$. Significance test of equality between relative risk values: $p=4.5 \times 10^{-5}$. Annual increase according to regressionline, $3 \cdot 5 \%$.

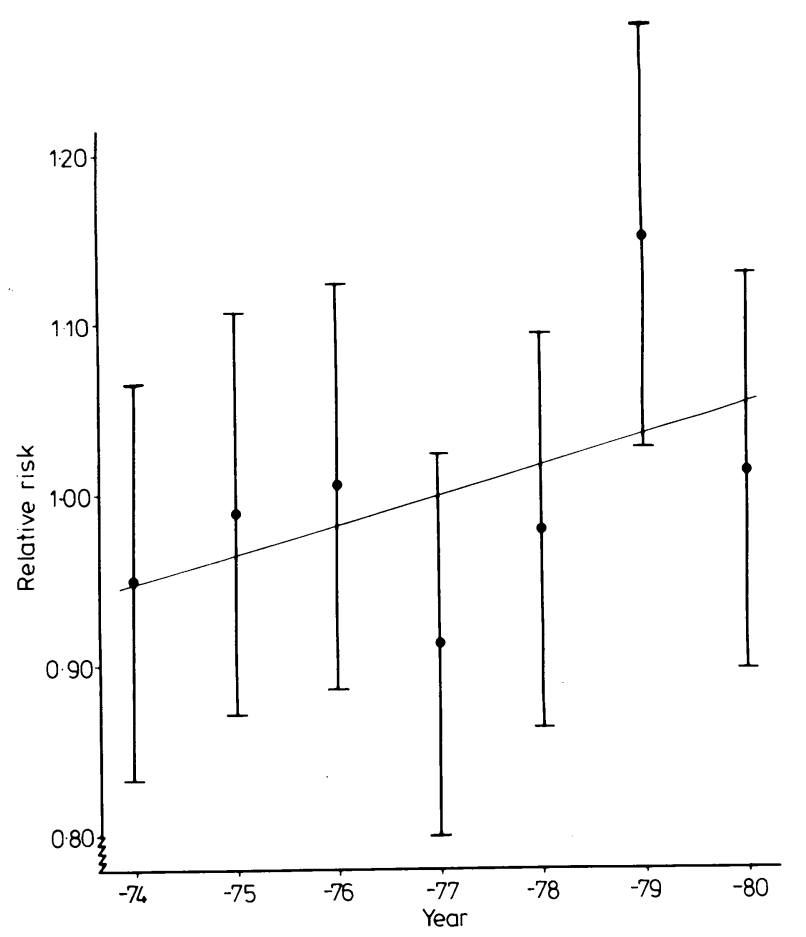

FIG 4-Mortality from myocardial infarction among women aged 40-74 Relative risks for specific years according to the multiplicative model and regression line. Test of model: $p=0 \cdot 9$. Significance test of equality between relative risk values: $p \quad 0 \cdot 16$. Annual increase according to regression line $1 \cdot 7 \%$.

This study used data obtained from the national register of deaths and from a medical information system. The validity of this information together with the methodology for using it for epidemiological purposes has been described. ${ }^{8}$ In general this information was found to be as accurate as that obtained fromk special registers of myocardial infarction. Despite this one explanation of the observed trend may have been an increase tendency of doctors during the period to make the diagnosis of. myocardial infarction or an increased tendency of patients to? 
seek medical care for symptoms that may have been due to a myocardial infarction. ${ }^{12}$ If so, an increase in the proportion of less severe cases would have been expected. Since the mortality increased as much as the incidence, however, this seems to be a less likely explanation. Furthermore, a decrease in lethality might also have been expected. A scrutiny of case fatality rates, however, showed no such decrease.

The increases in incidence and mortality rates appeared to be real and were probably not due to an increasing tendency to seek hospital treatment or to make the diagnosis. The reason for the increase remains unknown. As regards treatment, the proportion of patients with treated and well controlled hypertension seems to have risen. ${ }^{13}$ Nevertheless, we do not know whether the prevalence of hypertension increased during the period. The use of beta-blockers increased considerably during the period. The effect of this on morbidity and mortality from myocardial infarction is, however, low. ${ }^{13} 14$ The effect of coronary bypass operations on the disease is probably negligible. ${ }^{13}$ It is also probable that the improvement, if any, in mortality rates due to the introduction of coronary care units occurred mainly before the period studied.

As regards risk indicators, a favourable trend is reflected in the decline in the proportion of smokers in the Swedish population during the period according to surveys carried out by the National Smoking and Health Association. An unfavourable trend, however, is the increase in the proportion of fat in the diet of the population during the past four decades at least according to official statistics. In Sweden the years 1974-80 were characterised by social and economic instability which was unknown to Swedes, who are accustomed to the idea of almost total social security. According to Brenner socioeconomic factors contributed to changes in mortality from coronary heart disease. ${ }^{15}$ Possible neuroendocrine mechanisms underlying these associations are, however, largely unknown. Further research is needed into the trends of known risk factors of cardiovascular disease.
This study was supported by grants from the Swedish Medical Research Council (project No 6216).

\section{References}

1 Gordon T, Thomas T. The recent decrease in CHD mortality. Prev Med $1975 ; 4: 115-25$.

2 Cooper R, Stamler J, Dyer A, Garside D. The decline in mortality from coronary heart disease, USA, 1968-1975. F Chron Dis 1978;31:709-20.

${ }^{3}$ World Health Organisation. Prevention of coronary heart disease. Technical report series No 678. Geneva: WHO, 1982.

4 Gillum R, Blackburn H, Feinleib M. Current strategies for explaining the decline in ischemic heart disease mortality. F Chron Dis 1982;35:467-74.

5 World Health Organisation. World health statistics. Geneva: WHO, 1978.

${ }^{6}$ Regional Office for Europe. Myocardial infarction registers. Public health in Europe 5. Copenhagen: WHO, 1976.

7 National Board of Health and Welfare. International statistical classification of diseases, injuries and causes of death. 1965 revision adapted for indexing of hospital records and morbidity statistics. 3 rd ed. Stockholm: Liber, 1973.

${ }^{8}$ Ahlbom A. Acute myocardial infarction in Stockholm. A medical information system as an epidemiological tool. Int $\mathcal{f}$ Epidemiol 1978;7:271-6.

9 Ahlbom A, Nordlander R. Application of diagnostic criteria in the diagnoses of myocardial infarction. Scand F Soc Med 1979;7:67-72.

${ }^{10}$ Breslow NE, Day NE. Indirect standardization and multiplicative models for rates, with reference to the age adjustment of cancer incidence and relative frequency data. F Chron Dis 1975;28:289-303.

11 Statistical Analysis Systems Institute. In: Helwig JT, Council KA, eds. $S A S$ user's guide, 1979. Cary, North Carolina: SASI, 1979.

12 Isacsson SO, Johansson B. Myocardial infarction in Malmö during the 10-year period 1963-72. Acta Med Scand 1979;202:293-8.

${ }^{13}$ de Faire U. Mortality from ischemic heart disease in relation to prescription of beta-blockers. In: Beta-adrenoceptor antagonist treatment of patients with myocardial infarction. Stockholm: Liber, 1983.

14 Rose G. The contribution of intensive coronary care. Br $\mathcal{F}$ Prev Soc Med $1975 ; 29: 147-50$.

15 Brenner $\mathrm{MH}$, Mooney A. Economic change and sex-specific cardiovascular mortality in Britain 1955-1976. Soc Sci Med 1982;16:431-42.

(Accepted 22 March 1983)

\section{SHORT REPORTS}

\section{Screening the newborn for Duchenne muscular dystrophy: parents' views}

Duchenne muscular dystrophy is a crippling, progressive, and ultimately fatal neuromuscular disease $\mathrm{It}$ is inherited as an $\mathrm{X}$ linked recessive trait in two thirds of cases, the remainder being new mutations. Although the disease is present at birth, it is often not diagnosed until the boy is at least 4 or 5 years old. One of the diagnostic features of the disease is an extremely high serum creatine kinase activity. A sufficiently sensitive method of measuring this activity that is generally agreed to be suitable for use in neonatal screening for the disease is available.' Pilot studies using this technique have been implemented in some countries, but ethical concerns have been expressed since there is no treatment that will benefit the sufferer. There is also concern regarding the psychological effects of early diagnosis. We asked parents of boys with the disease whether they were in favour of screening for the disease and, if they were, when they thought it should be carried out.

\section{Methods and results}

We interviewed 69 parents from 53 families of boys suffering from Duchenne muscular dystrophy to explore their views of neonatal screening, services, and their experiences at the time of diagnosis. ${ }^{2}$ All parents were asked their opinions on the desirability of screening for the disease and, if they were in favour of screening, whether it should be carried out close to birth, at 18 months, or at some other time.

In $75 \%$ of the families $(90 \%$ of those who expressed an opinion) the parents were in favour of neonatal screening. This finding is similar to the findings of Beckmann and Scheuerbrandt. ${ }^{3}$ Of the four parents who thought that screening should take place at some other time, three said it should be carried out at between 1 and 3 months. Three parents thought that in their case earlier diagnosis would have been undesirable.

Parents were also asked the reasons for their views. Five major reasons were given by those who stated a preference for neonatal screening: (1) it prevented parents experiencing the negative effects of long delays between the first symptoms and diagnosis that are associated with the present diagnostic system; (2) parents had a "right" to be informed as soon as scientifically possible; (3) it prevented the birth of further affected sufferers of the disease to carrier mothers and their female relatives; (4) it had practical advantages - for example, the opportunity to buy or rent appropriate housing; and (5) it had emotional advantages.

\section{Comment}

No control group was used in this study, and the views of the parents on the advantages and disadvantages of neonatal screening were not based on any experience of such screening. The parents had, however, experienced a diagnostic system that many had found unsatisfactory.

Two major ethical objections to neonatal screening have been raised: firstly, there is no treatment that will benefit the patient; and, secondly, diagnosis might have psychological effects, including rejection or overprotection of the child. Although no cure exists, there is an argument that with a disease as devastating as Duchenne muscular dystrophy "the family is the patient." 4 The potential benefits of neonatal screening to families include early support and genetic counselling. Two important influences on how parents react to the diagnosis of a serious disease are the way in which they are told of the diagnosis and how they are supported subsequently. Our study disclosed dissatisfaction with both these points. ${ }^{5}$ Our findings mirror those of studies of other handicapping conditions, which 